\title{
Utility of Administrative Databases and Big Data on Understanding Glioma Treatment-A Systematic Review
}

\author{
Monica-Rae Owens ${ }^{1}$ Sarah Nguyen ${ }^{1}$ Michael Karsy ${ }^{2}$ \\ ${ }^{1}$ Department of Neurosurgery, University of Utah, Utah, United \\ States \\ Address for correspondence Michael Karsy, MD, PhD, MSc, University \\ of Utah Health Care, University of Utah Health Hospitals and Clinics, \\ 2 University of Utah Health Care, University of Utah Health Hospitals \\ UT, United States (e-mail: michael.karsy@hsc.utah.edu). \\ and Clinics, Utah, United States \\ Indian J Neurosurg 2022;11:104-117.
}

\begin{abstract}
Background Gliomas are a heterogeneous group of tumors where large multicenter clinical and genetic studies have become increasingly popular in their understanding. We reviewed and analyzed the findings from large databases in gliomas, seeking to understand clinically relevant information.

Methods A systematic review was performed for gliomas studied using large administrative databases up to January 2020 (e.g., National Inpatient Sample [NIS], National Surgical Quality Improvement Program [NSQIP], and Surveillance, Epidemiology, and End Results Program [SEER], National Cancer Database [NCDB], and others).

Results Out of 390 screened studies, 122 were analyzed. Studies included a wide range of gliomas including low- and high-grade gliomas. The SEER database $(n=83)$ was the most used database followed by $\operatorname{NCDB}(n=28)$. The most common pathologies included glioblastoma multiforme (GBM) $(n=67)$, with the next category including mixes of grades II to IV glioma $(n=31)$. Common study themes involved evaluation of descriptive epidemiological trends, prognostic factors, comparison of different pathologies, and evaluation of outcome trends over time. Persistent health care disparities in patient outcomes were frequently seen depending on race, marital status, insurance status, hospital volume, and location, which did not change over time. Most studies showed improvement in survival because of advances in surgical and adjuvant treatments.
Keywords
Conclusions This study helps summarize the use of clinical administrative databases
- administrative in gliomas research, informing on socioeconomic issues, surgical outcomes, and database
- glioma
- glioblastoma adjuvant treatments over time on a national level. Large databases allow for some study questions that would not be possible with single institution data; however, limitations remain in data curation, analysis, and reporting methods.

DOI https://doi.org/ $10.1055 / s-0042-1742333$ ISSN 2277-954X.

\footnotetext{
(c) 2022. Neurological Surgeons' Society of India. All rights reserved. This is an open access article published by Thieme under the terms of the Creative Commons Attribution-NonDerivative-NonCommercial-License, permitting copying and reproduction so long as the original work is given appropriate credit. Contents may not be used for commercial purposes, or adapted, remixed, transformed or built upon. (https://creativecommons.org/ licenses/by-nc-nd/4.0/)

Thieme Medical and Scientific Publishers Pvt. Ltd., A-12, 2nd Floor, Sector 2, Noida-201301 UP, India
} 


\section{Introduction}

Gliomas encompass the second most common type of brain tumor in the United States, while glioblastoma multiforme (GBM) accounts for the most common malignant primary brain tumor. ${ }^{1}$ Multiple advances in treatment, including earlier imaging and detection, safer surgical resection, and adjuvant radiochemotherapy (RCT) have improved disease treatment. $^{2}$ Clinical variables, such as age and surgical resection, in addition to genetic factors have been helpful in stratifying patient risk. However, there remains heterogeneity in the outcomes of patients and treatment response.

The rise in the use of administrative databases and big data has been notable in neurosurgery, but their impact on glioma understanding remains limited. ${ }^{3}$ These studies often show multiple associative findings due to their significant sample sizes without distinction between statistical and clinical significance. Moreover, the clinical impact of these studies remains unclear. The purpose of this review was to accumulate and compare the lessons learned from big data regarding gliomas and identify future challenges for exploration.

\section{Methods}

We aimed to evaluate the impact of multicenter, publicly available administrative databases on clinically relevant information in the treatment of gliomas. A literature search of PubMed was performed using the following search terms: (National Surgical Quality Improvement Program OR NSQIP OR National Inpatient Sample OR NIS OR HCUP-NIS OR Kid's Inpatient Database OR HCUP-KID OR Surveillance, Epidemiology, and End Results Program OR SEER OR Pediatric Health Information Systems OR PHIS OR MarketScan OR Administrative database OR SEER OR SEERMedicare OR CBTRUS OR NCDB OR NRD OR SID OR SASD OR CMS OR Vizient OR Premier OR PearlDiver OR Optum) AND (glioma OR glioblastoma OR astrocytoma OR oligodendroglioma OR anaplastic astrocytoma OR anaplastic oligodendroglioma [AO]). References from manuscripts were also reviewed to identify relevant studies.

Studies up to January 2020 and English-only manuscripts were included. Studies were reviewed independently by two reviewers (M.O., M.K.) to exclude case reports, reviews, and laboratory studies. The main study findings including prognostic factors, sample number, database type, World Health Organization (WHO) tumor status, tumor types, surgical complications, and surgical outcomes were noted. Papers were narrowed to those discussing low-grade glioma (LGG), including grade II gliomas, astrocytoma and oligodendroglioma, as well as high-grade gliomas (HGG), including anaplastic astrocytoma (AA), anaplastic oligodendroglioma (AO), and GBM. Studies used a combination of histological and molecular classification, depending on the year of study. Several studies incorporated other glioma types that were excluded (Supplemental discussion, -Fig. S1, -Table S1). The Preferred Reporting
Items for Systematic Reviews and Meta-Analyses (PRISMA) guidelines were used to draft this manuscript. Descriptive statistics are demonstrated where relevant.

\section{Results}

A total of 390 studies were screened and narrowed down to 122 studies ( - Fig. 1 ). Studies included AA only $(n=2)$, AA with other pathologies $(n=3)$, AO only $(n=2), \operatorname{GBM}(n=67)$, grades II to IV glioma $(n=31)$, grade II glioma $(n=3)$, HGG $(n=7), \quad$ mix of high-grade tumors $(n=1)$, and oligodendroglioma $(n=6)(-$ Table 1$)$. The most common databases included the NCDB $(n=28)$ and SEER databases $(n=83)$. A further breakdown of all studies into LGG and HGG was performed (-Figs. S1-53).

\section{Low Grade Glioma}

LGGs were evaluated as an aggregate group in three studies. One study on grade II gliomas showed that RCT did not improve survival compared with chemotherapy alone, ${ }^{4}$ and two others showed that greater extent of resection (EOR) improved prognosis., ${ }^{5,6}$ Unfortunately, these studies were limited by aggregating all LGGs, which often behave distinctly.

Six studies specifically evaluated oligodendroglioma, ${ }^{7-12}$ with two of these studies showing improved survival over the preceding 10 years (e.g., 2004-2013) mainly as a function of improved surgery and RCT. ${ }^{9,12}$ Achey et al evaluated the age-adjusted incidence rate from the Central Brain Tumor Registry of the United States from 2000 to $2013 .^{7}$ A decreased incidence of oligodendroglioma and AO was seen, although this was possibly because of recent changes in molecular classification. Prognostic factors impacting survival for oligodendrogliomas in other studies included age, sex, race, and EOR. ${ }^{8,10-12}$ Alattar et al evaluated 3,406 patients with oligodendroglioma from 1999 to 2010 using the SEER database and demonstrated an improvement in OS after gross-total resection compared with AA or GBM. ${ }^{8}$ This finding was confirmed by Kinslow et al in their evaluation of 3135 cases from the SEER database data from 2004 to 2013; they also showed that EOR impacted survival in both oligodendroglioma and $\mathrm{AO}{ }^{10}$

Multiple studies have evaluated glioma by including either LGG as a broad category or comparing LGG with HGG. ${ }^{1,13-37}$ Several studies evaluated the epidemiology of gliomas and demonstrated increased likelihood in Caucasians, older, or male patients, patients with prior smoking history, women with prior breast cancer treatment, and insured patients. $^{13-16,19}$ Plascak et al evaluated 24,230 glioma patients using the SEER database between 2000 and 2006; they showed a greater incidence of gliomas in countries with higher socioeconomic status, which suggested unequal distribution of diagnostic resources. ${ }^{19}$ Another study using the SEER database noted a decreasing incidence of gliomas and suggested there was a shift to increased diagnosis of GBM by pathological criteria over time. ${ }^{18}$ The vast majority of studies involving glioma have looked at prognostic factors. These studies have suggested that White race, younger age, more 


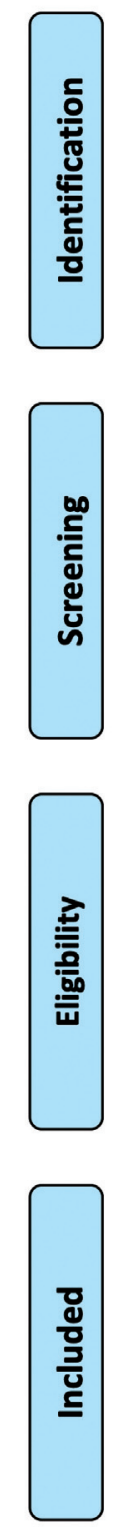

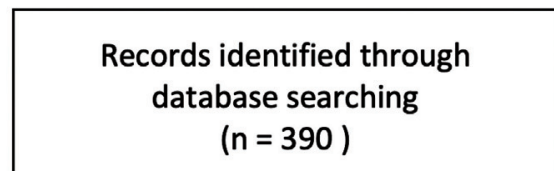

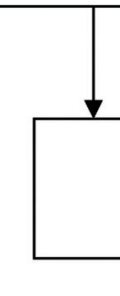

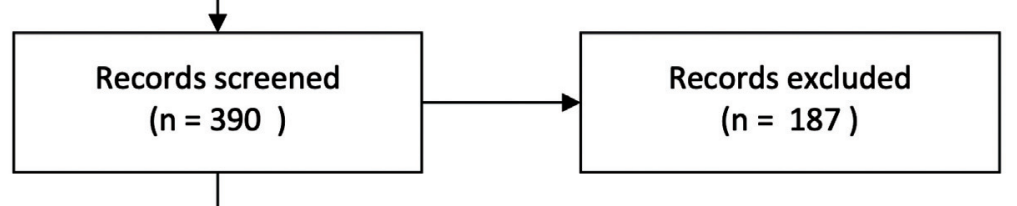

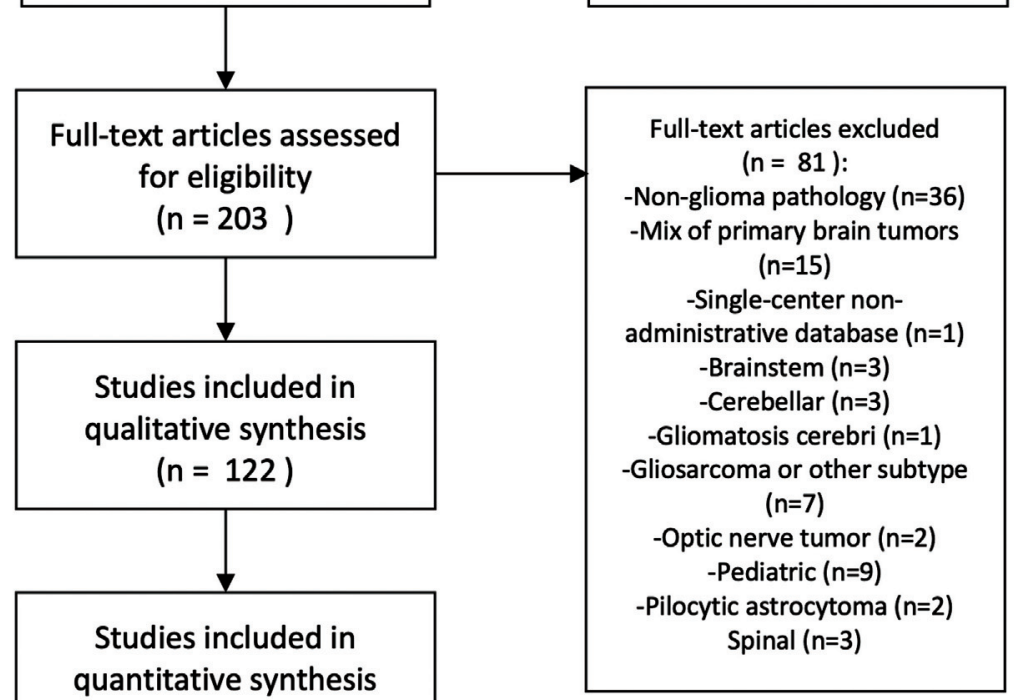

(meta-analysis)

$(n=122)$

Fig. 1 The Preferred Reporting Items for Systematic reviews and Meta-Analyses (PRISMA) flow diagram of selected studies.

recent diagnosis, lower WHO grade or histology, marital status, better socioeconomic status, EOR, radiotherapy, radiochemotherapy, treatment at high-volume facilities, and tumor size positively impact patient prognosis. ${ }^{1,4,16,17,20-36}$ Missios et al evaluated 21,384 cases of glioma between 2005 and 2011 using the National Inpatient Sample (NIS) database and showed that perioperative complications were increased by greater age, coagulopathy, coronary artery disease, congestive heart failure, and smoking history. ${ }^{37}$

Although these studies have had an impact by identifying consistent risk and prognostic factors, they are limited by aggregating glioma types, mostly as a limitation of lacking molecular data in nearly all multicenter databases. Moreover, both epidemiology and prognostic factor studies suggest geographic differences in outcomes because of medical resources and facilities but do little to address these inequalities or report in sufficient detail to confer actionable insight. Despite improve standardization of glioma treatment with clinical guidelines, treatment variation likely still occurs, which is impacted by patient socioeconomics and remains poorly understood.

\section{High-Grade Glioma}

Several studies have evaluated and compared outcomes for AA and AO. Prognostic factors for AA included age, RCT, private insurance, higher income, tumor site, marital status, EOR, histology, and treatment with RT. ${ }^{38-44}$ Smoll et al specifically looked at 3,202 patients with AA between 1973 and 2006 in the SEER database, showing worsened mortality compared with matched controls. ${ }^{43}$ A 5- and 10year overall survival (OS) of $23.6 \%$ and $15.1 \%$ was identified, respectively. Age significantly impacted prognosis, however, improvement in survival was not seen over the study time period. In contrast, Shin et al evaluated 1,692 patients with 
Table 1 Studies evaluating administrative studies/big data in LGG and HGG

\begin{tabular}{|c|c|c|c|c|c|}
\hline $\begin{array}{l}\text { Database } \\
\text { used }\end{array}$ & $\begin{array}{l}\text { Patient } \\
\text { pathology }\end{array}$ & $\begin{array}{l}\text { Sample } \\
\text { size }\end{array}$ & Study year & Study findings & Reference \\
\hline NCDB & $\mathrm{AA}$ & 4,807 & $2004-2013$ & $\begin{array}{l}\text { Prognostic factors: } R C T \text {, age, private insur- } \\
\text { ance, higher income }\end{array}$ & $\operatorname{Shin}^{40}$ \\
\hline SEER & $\mathrm{AA}$ & 3,202 & $1973-2006$ & $\begin{array}{l}\text { 5-year OS 23.6\%; prognostic factor: age } \\
\text { (only } 3 \text { years postdiagnosis); } 5 \text { year survival } \\
\text { unchanged over time }\end{array}$ & $\mathrm{Smoll}^{43}$ \\
\hline SEER & $\mathrm{AA}, \mathrm{AO}$ & 1,939 & $1973-2013$ & $\begin{array}{l}\text { Prognostic factors: age, tumor site, marital } \\
\text { status, EOR, histology, RT, surgery }\end{array}$ & Zhao $^{44}$ \\
\hline SEER & $\mathrm{AA}, \mathrm{AO}$ & 390 & $1990-2008$ & $\begin{array}{l}\text { Evaluated patients }>70 \text { years of age; me- } \\
\text { dian OS } 11 \text { month; prognostic factors: EOR, } \\
\text { RT, gender, marital status }\end{array}$ & Mukherjee $^{38}$ \\
\hline SEER & $\mathrm{AA}, \mathrm{GBM}$ & 24717 & $1999-2010$ & $\begin{array}{l}\text { Median OS (AA versus GBM with GTR) } 64 \\
\text { versus } 13 \text { months; prognostic factors: EOR }\end{array}$ & Padwal $^{39}$ \\
\hline NCDB & $\mathrm{AO}$ & 1,643 & $2004-2013$ & Prognostic factors: race, age, RCT & $\operatorname{Shin}^{42}$ \\
\hline NCDB & $\mathrm{AO}$ & 1,692 & $2004-2013$ & $\begin{array}{l}\text { 5-year OS 59.8\%; prognostic factors: RCT, } \\
\text { single-agent chemotherapy }\end{array}$ & $\operatorname{Shin}^{41}$ \\
\hline $\mathrm{NCDB}$ & GBM & 100,672 & $1998-2011$ & $\begin{array}{l}\text { Median OS } 7.5 \text { months; prognostic factor: } \\
\text { age, tumor histology, race, gender, educa- } \\
\text { tion, insurance status, EOR, RCT, tumor } \\
\text { location }\end{array}$ & Dressler $^{56}$ \\
\hline NCDB & GBM & 60,672 & $2004-2013$ & $\begin{array}{l}\text { Median OS } 8.1 \text { months; prognostic factors: } \\
\text { TMZ, high volume facility treatment; } 2- \\
\text { month survival benefit in high-volume } \\
\text { centers }\end{array}$ & Aulakh ${ }^{55}$ \\
\hline NCDB & GBM & 738 & $2010-2012$ & $\begin{array}{l}\text { Median OS (RCT) } 15.6 \text { months, 2-year OS } \\
25.9 \% \text {; limited benefit of RT in patients with } \\
\text { MGMT methylation }\end{array}$ & $\operatorname{Lee}^{60}$ \\
\hline NCDB & GBM & 448 & $2010-2013$ & $\begin{array}{l}\text { Median OS (RCT) } 8.7 \text { months; prognostic } \\
\text { factors: age, EOR, RCT; }\end{array}$ & Malakhov ${ }^{59}$ \\
\hline NCDB & GBM & 4,598 & $1998-2011$ & $\begin{array}{l}\text { Prognostic factor: long-course radiothera- } \\
\text { py compared with short-course } \\
\text { radiotherapy }\end{array}$ & $\mathrm{Mak}^{58}$ \\
\hline NCDB & GBM & 114,979 & $1998-2012$ & $\begin{array}{l}\text { Prognostic factor: RCT compared with ra- } \\
\text { diation alone }\end{array}$ & Glaser $^{57}$ \\
\hline NCDB & GBM & 114,979 & $1998-2012$ & $\begin{array}{l}\text { Prognostic factors: disparities in standard } \\
\text { of care secondary to race, insurance status } \\
\text { and institution of treatment }\end{array}$ & Rhome $^{65}$ \\
\hline $\mathrm{NCDB}$ & GBM & 5,252 & $2004-2012$ & $\begin{array}{l}\text { Prognostic factor: single-agent chemo- } \\
\text { therapy with radiotherapy in elderly } \\
\text { patients }\end{array}$ & Huang ${ }^{111}$ \\
\hline $\mathrm{NCDB}$ & GBM & 89,839 & $2004-2013$ & $\begin{array}{l}\text { Prognostic factor: patients treated in } \\
\text { academic/research programs, high-volume } \\
\text { centers }\end{array}$ & Hauser $^{64}$ \\
\hline NCDB & GBM & 27,865 & $2004-2013$ & $\begin{array}{l}\text { Prognostic factor: GTR; no survival benefit } \\
\text { of STR over biopsy }\end{array}$ & Trifiletti $^{67}$ \\
\hline NCDB & GBM & 1,223 & $2004-2014$ & $\begin{array}{l}\text { Dose escalation not associated with sur- } \\
\text { vival; prognostic factors: age, comorbidity } \\
\text { score, hospital volume (noncommunity } \\
\text { centers), education level, income, insur- } \\
\text { ance status, race, gender }\end{array}$ & Wegner $^{68}$ \\
\hline NCDB & GBM & 45,942 & 2004-2015 & $\begin{array}{l}\text { Prognostic factor: younger age, female } \\
\text { gender, black ethnicity, higher KPS, and } \\
\text { GTR over STR; delay }>8 \text { weeks for radio- } \\
\text { chemotherapy detrimental after GTR; de- } \\
\text { lay }<4 \text { weeks for radiochemotherapy } \\
\text { detrimental after STR }\end{array}$ & Buszek $^{62}$ \\
\hline NCDB & GBM & 45,268 & 2004-2016 & $\begin{array}{l}\text { Median OS was } 12.8 \text { months vs } 8.3 \text { months } \\
\text { for patients with unifocal GBM or multifocal }\end{array}$ & Haque $^{63}$ \\
\hline
\end{tabular}


Table 1 (Continued)

\begin{tabular}{|c|c|c|c|c|c|}
\hline $\begin{array}{l}\text { Database } \\
\text { used }\end{array}$ & $\begin{array}{l}\text { Patient } \\
\text { pathology }\end{array}$ & $\begin{array}{l}\text { Sample } \\
\text { size }\end{array}$ & Study year & Study findings & Reference \\
\hline & & & & $\begin{array}{l}\text { GBM; prognostic factor: radiochemother- } \\
\text { apy; radiochemotherapy beneficial even if } \\
\text { multifocal }\end{array}$ & \\
\hline NCDB & GBM & 13,489 & 2005-2012 & $\begin{array}{l}\text { Prognostic factors: delay in radiochemo- } \\
\text { therapy treatment }\end{array}$ & Yusuf $^{76}$ \\
\hline NCDB & GBM & 1,479 & 2006-2011 & $\begin{array}{l}\text { Prognostic factor: radiochemotherapy } \\
\text { compared with radiation alone }\end{array}$ & Kole 66 \\
\hline NCDB & GBM & 12,725 & 2010-2012 & $\begin{array}{l}\text { Median OS (MGMTme versus MGMT-) } 20 \\
\text { versus } 15 \text { months; prognostic factors: RCT } \\
\text { in MGMTme tumors }\end{array}$ & Lee $^{61}$ \\
\hline NCDB; RTOG & GBM & 40,396 & 1974-2002 & $\begin{array}{l}\text { Survival for patients in the RTOG database } \\
\text { exceeded survival in patients in the NCDB } \\
\text { group likely because patients in the RTOG } \\
\text { database come from clinical trials which } \\
\text { have specific enrollment criteria; prognos- } \\
\text { tic factor: age }\end{array}$ & Siker $^{73}$ \\
\hline SEER & GBM & 9,103 & 1973-2006 & $\begin{array}{l}\text { Prognostic factor: age, marital status, } \\
\text { resection }\end{array}$ & Walker $^{75}$ \\
\hline SEER & GBM & 21,783 & $1973-2007$ & Prognostic factors: EOR, RT & Zinn $^{77}$ \\
\hline SEER & GBM & 34,664 & $1973-2008$ & $\begin{array}{l}\text { Prognostic factor: race (Asian/Pacific Is- } \\
\text { lander), surgical resection, age, RT; im- } \\
\text { proved survival over time }\end{array}$ & Thumma $^{74}$ \\
\hline SEER & GBM & 51,518 & $1973-2014$ & Prognostic factor: no prior history of cancer & Al-Husseini ${ }^{70}$ \\
\hline SEER & GBM & 60,456 & $1973-2015$ & $\begin{array}{l}\text { Prognostic factors: age, tumor size, tumor } \\
\text { location, GTR, radiation, chemotherapy, } \\
\text { brachytherapy }\end{array}$ & Bartek $^{72}$ \\
\hline SEER & GBM & 25,117 & $1985-2014$ & $\begin{array}{l}\text { Prognostic factors: Hispanic Latino (GBM } \\
\text { and GBM-GC), age, gender }\end{array}$ & Bin Abdulrahman ${ }^{120}$ \\
\hline SEER & GBM & 10,987 & $1988-2001$ & Prognostic factors: marital status & Chang $^{78}$ \\
\hline SEER & GBM & 1,530 & 1991-1999 & Prognostic factor: race & Barnholtz-Sloan 71 \\
\hline SEER & GBM & 1,375 & 1991-2002 & $\begin{array}{l}\text { Median time post-resection to initiation of } \\
\text { RT was } 15 \text { days; no impact of wait time on } \\
\text { OS }\end{array}$ & Lai $^{121}$ \\
\hline SEER & GBM & 1,273 & $1991-2007$ & $\begin{array}{l}\text { No difference in patient outcomes between } \\
\text { low- and high-readmission rate hospitals }\end{array}$ & Nuno ${ }^{117}$ \\
\hline SEER & GBM & 19,674 & 1993-2007 & Improved survival over time & Darefsky ${ }^{80}$ \\
\hline SEER & GBM & 4,137 & 1994-2002 & $\begin{array}{l}\text { Prognostic factors: age, marital status, and } \\
\text { comorbidities }\end{array}$ & Iwamoto 81 \\
\hline SEER & GBM & 1,652 & 1995-2009 & $\begin{array}{l}\text { Median OS of } 7.4,5.9 \text { and } 5.6 \text { months for } \\
\text { TMZ/RT, RT alone (2005-2009) and RT } \\
\text { alone (1995-1999), respectively; benefit of } \\
\text { TMZ addition to RT in later years }\end{array}$ & Arvold $^{98}$ \\
\hline SEER & GBM & 3,963 & 1997-2010 & $\begin{array}{l}\text { GTR supported as initial treatment and for } \\
\text { recurrence }\end{array}$ & Chen $^{122}$ \\
\hline SEER & GBM & 3,784 & $1997-2010$ & $\begin{array}{l}\text { Patients with postop infections showed no } \\
\text { significant difference in survival }\end{array}$ & Chen $^{123}$ \\
\hline SEER & GBM & 22,777 & 1998-2007 & $\begin{array}{l}\text { Factors associated with omission of RT } \\
\text { included older age, lower annual income, } \\
\text { African-American race, Hispanic race, } \\
\text { Asian-American race, unmarried status, } \\
\text { and STR/Bx; use of radiation associated } \\
\text { with improved OS }\end{array}$ & Aizer $^{69}$ \\
\hline SEER & GBM & 10,022 & $1998-2008$ & $\begin{array}{l}\text { Patients surviving past } 2 \text { years have favor- } \\
\text { able conditional probability of survival }\end{array}$ & Johnson $^{83}$ \\
\hline SEER & GBM & 20,705 & 1998-2009 & & Noorbakhsh $^{86}$ \\
\hline
\end{tabular}


Table 1 (Continued)

\begin{tabular}{|c|c|c|c|c|c|}
\hline $\begin{array}{l}\text { Database } \\
\text { used }\end{array}$ & $\begin{array}{l}\text { Patient } \\
\text { pathology }\end{array}$ & $\begin{array}{l}\text { Sample } \\
\text { size }\end{array}$ & Study year & Study findings & Reference \\
\hline & & & & $\begin{array}{l}\text { Benefit of 2-3 months survival after GTR in } \\
\text { all age groups; lower rate of GTR in older } \\
\text { patients }\end{array}$ & \\
\hline SEER & GBM & 342 & $1998-2009$ & $\begin{array}{l}\text { Median OS } 12 \text { months; prognostic factors: } \\
\text { EOR }\end{array}$ & Adams $^{82}$ \\
\hline SEER & GBM & 6,039 & $1999-2010$ & $\begin{array}{l}\text { Prognostic factors: gender (female), mar- } \\
\text { ried patients, ethnicity }\end{array}$ & Shah $^{89}$ \\
\hline SEER & GBM & 13,932 & $2000-2008$ & Benefit of TMZ to survival over time & Johnson $^{84}$ \\
\hline SEER & GBM & 6,586 & $2000-2008$ & Prognostic factor: gender (female) & $\operatorname{Tian}^{90}$ \\
\hline SEER & GBM & 20,879 & $2000-2009$ & $\begin{array}{l}\text { Benefit of TMZ and BZM to survival over } \\
\text { time }\end{array}$ & Wachtel $^{91}$ \\
\hline SEER & GBM & 302 & $2000-2010$ & $\begin{array}{l}\text { Prognostic factors: supratentorial location, } \\
\text { GTR, and later year of diagnosis }\end{array}$ & $\operatorname{Lam}^{85}$ \\
\hline SEER & GBM & 14,675 & $2000-2010$ & $\begin{array}{l}\text { Median OS } 11 \text { months; prognostic factors: } \\
\text { age, gender, marriage status, race (non- } \\
\text { Hispanics), region (northeast), nonlateral- } \\
\text { izing, small }(<3 \mathrm{~cm}) \text {, adjuvant radiation }\end{array}$ & $\operatorname{Pan}^{87}$ \\
\hline SEER & GBM & 26,481 & $2000-2010$ & $\begin{array}{l}\text { Prognostic factors: age, gender, race (non- } \\
\text { White), socioeconomic status }\end{array}$ & Porter ${ }^{88}$ \\
\hline SEER & GBM & 28,933 & $2000-2013$ & $\begin{array}{l}\text { Improved survival with TMZ with radiation } \\
\text { and adjuvant TMZ and then BEV after FDA } \\
\text { approval }\end{array}$ & Zhu $^{92}$ \\
\hline SEER & GBM & 20,550 & $2000-2013$ & $\begin{array}{l}\text { Prognostic factors: tumor size }(<5 \mathrm{~cm}) \text {, } \\
\text { EOR, RCT }\end{array}$ & Shu $^{96}$ \\
\hline SEER & GBM & 6,919 & $2001-2006$ & Benefit of TMZ to survival over time & Koshy ${ }^{94}$ \\
\hline SEER & GBM & 11,189 & $2001-2006$ & $\begin{array}{l}\text { Cure fraction of } 12 \% \text { for young adults at } 10 \\
\text { years }\end{array}$ & Smoll| ${ }^{97}$ \\
\hline SEER & GBM & 21,184 & $2001-2011$ & $\begin{array}{l}\text { Prognostic factors: race (Latinos); possibly } \\
\text { greater incidence of GBM-GC in Latinos }\end{array}$ & Shabihkhani ${ }^{95}$ \\
\hline SEER & GBM & 5,991 & $2004-2008$ & $\begin{array}{l}\text { Prognostic factors: age, marital status, } \\
\text { median income; factors led to increased } \\
\text { GTR and RT }\end{array}$ & Aneja $^{93}$ \\
\hline SEER & GBM & 24,262 & $2004-2013$ & $\begin{array}{l}\text { Regional differences in survival and inci- } \\
\text { dence in the US; prognostic factors: age, } \\
\text { marital status, race, tumor laterality, WHO } \\
\text { grade, disease extent, tumor size, tumor } \\
\text { extension, RCT }\end{array}$ & $\mathrm{Xu}^{119}$ \\
\hline SEER & GBM & 24,348 & $2004-2013$ & $\begin{array}{l}\text { Median survival } 15,15 \text { and } 5 \text { months for } \\
\text { pediatric, adult, and elderly, respectively }\end{array}$ & $\mathrm{Li}^{116}$ \\
\hline SEER & GBM & 30,767 & $2004-2015$ & $\begin{array}{l}\text { Prognostic factors: marriage, age, race, } \\
\text { middle-income counties }\end{array}$ & $\mathrm{Xie}^{52}$ \\
\hline SEER & GBM & 5,607 & $2006-2010$ & $\begin{array}{l}\text { Median OS of } 8,7 \text {, and } 9 \text { months in } 2006 \text {, } \\
2008 \text {, and 2010, respectively; improved } \\
\text { survival with BZM }\end{array}$ & Johnson $^{105}$ \\
\hline SEER & GBM & 2,603 & $2006-2011$ & Benefit of BZM to survival & Davies $^{102}$ \\
\hline SEER & GBM & 13,665 & $2007-2012$ & $\begin{array}{l}\text { Prognostic factors: insurance status, RT, } \\
\text { marital status; improved OS over time }\end{array}$ & Rong $^{110}$ \\
\hline SEER & GBM & 3,473 & $2010-2014$ & $\begin{array}{l}\text { Prognostic factors: race (Asian-Pacific } \\
\text { Islander) }\end{array}$ & Bohn $^{101}$ \\
\hline \multirow{2}{*}{$\begin{array}{l}\text { SEER; Broad Institute } \\
\text { Genotype Tissue Ex- } \\
\text { pression project; UCSF } \\
\text { 10K Immunomes- } \\
\text { database }\end{array}$} & GBM & & $1973-2016$ & $\begin{array}{l}\text { Increased expression of immunoregulatory } \\
\text { molecules in the elderly }\end{array}$ & Ladomersky $^{106}$ \\
\hline & GBM & 5,029 & 1999-2007 & Median survival was 4.9 months & Arvold $^{99}$ \\
\hline
\end{tabular}


110 Utility of Administrative Databases and Big Data on Understanding Glioma Treatment Owens et al.

Table 1 (Continued)

\begin{tabular}{|c|c|c|c|c|c|}
\hline $\begin{array}{l}\text { Database } \\
\text { used }\end{array}$ & $\begin{array}{l}\text { Patient } \\
\text { pathology }\end{array}$ & $\begin{array}{l}\text { Sample } \\
\text { size }\end{array}$ & Study year & Study findings & Reference \\
\hline \multicolumn{6}{|l|}{$\begin{array}{l}\text { SEER; Medicare } \\
\text { database }\end{array}$} \\
\hline ACS-NSQIP & GBM & 1,016 & $2012-2016$ & $\begin{array}{l}\text { Patients' aged } 65-89 \text { years included; } 3.4 \% \\
30 \text {-day death rate; } 5.7 \% \text { severe complica- } \\
\text { tion rate; } 33 \% \text { change in living disposition } \\
\text { rate }\end{array}$ & Rahmani $^{109}$ \\
\hline $\begin{array}{l}\text { California Office of } \\
\text { Statewide Health Plan- } \\
\text { ning \& Development } \\
\text { inpatient-discharge ad- } \\
\text { ministrative database }\end{array}$ & GBM & 18,506 & 1995-2010 & $\begin{array}{l}13.2 \% 30 \text {-day readmission rate; each read- } \\
\text { mission represented an additional } \$ 20,296 \\
\text { in median hospital charges on top of the } \\
\$ 72,029 \text { in charges for the index neuro- } \\
\text { surgical admission }\end{array}$ & Marcus $^{107}$ \\
\hline MarketScan & GBM & 14,037 & 2007-2016 & $\begin{array}{l}\text { Functional mapping associated with de- } \\
\text { creased complications, reoperations, } \\
\text { emergency department visits, and shorter } \\
\text { lengths of stay; no difference in charges } \\
\text { with functional versus no mapping }\end{array}$ & Pendharkar $^{108}$ \\
\hline SEER & GBM & 48748 & $2000-2013$ & $\begin{array}{l}\text { Multiple primary tumors associated with } \\
\text { female gender, White race, smaller tumors }\end{array}$ & Nguyen $^{112}$ \\
\hline SEER & GBM & 12,437 & $2002-2011$ & $\begin{array}{l}\text { Hospice enrollment associated with older } \\
\text { age, female gender, higher education, } \\
\text { White race, lower median income }\end{array}$ & Forst $^{104}$ \\
\hline $\begin{array}{l}\text { Los Angeles County } \\
\text { Cancer Surveillance } \\
\text { Program, CCR, and SEER }\end{array}$ & GBM & 38,567 & 1996-2006 & $\begin{array}{l}\text { Increased incidence of frontal, temporal } \\
\text { and cerebellar GBM over time compared } \\
\text { with other locations }\end{array}$ & Zada $^{113}$ \\
\hline SEER & GBM & 21,493 & 1973-1997 & Prognostic factor: race (white) & Barnholtz-Sloan 100 \\
\hline SEER & GBM & 1,181 & $1973-2015$ & $\begin{array}{l}\text { Prognostic factors (for secondary malig- } \\
\text { nancy): age, race, differentiated grade of } \\
\text { cancer tissue, marital status, WHO grade, } \\
\text { latency }\end{array}$ & Wang $^{118}$ \\
\hline SEER & GBM & 37,581 & $2001-2011$ & $\begin{array}{l}\text { Median OS } 14 \text { versus } 11 \text { months of metro- } \\
\text { politan versus non-metropolitan; prognos- } \\
\text { tic factors: urban area }\end{array}$ & Delavar $^{103}$ \\
\hline FCDS & Glioma & 14,092 & $1981-2013$ & $\begin{array}{l}\text { Diagnostic factor: race (Caucasian more } \\
\text { likely), age (older), gender (male), smoking } \\
\text { status, insurance status }\end{array}$ & Persaud-Sharma ${ }^{15}$ \\
\hline NCDB & Glioma & 49,405 & $2004-2013$ & $\begin{array}{l}\text { Proton beam therapy better median and 5- } \\
\text { year OS compared with other radiation } \\
\text { therapy }\end{array}$ & Jhaveri ${ }^{17}$ \\
\hline NCDB & Glioma & 5,036 & $2010-2014$ & Prognostic factors: female gender (GBMs) & Gittleman $^{20}$ \\
\hline NIS & Glioma & 21,384 & $2005-2011$ & $\begin{array}{l}\text { Identified perioperative complication risk: } \\
\text { age, coagulopathy, CAD, CHF, smoking }\end{array}$ & Missios $^{37}$ \\
\hline SEER & Glioma & 5,956 & $1973-2010$ & Prognostic factor: race (White) & Samaan $^{21}$ \\
\hline SEER & Glioma & 49,124 & $1973-2014$ & $\begin{array}{l}\text { Increased incidence of GBM versus non- } \\
\text { GBM over time }\end{array}$ & $\mathrm{Li}^{18}$ \\
\hline SEER & Glioma & 389,415 & $1973-2014$ & $\begin{array}{l}\text { Increased risk of gliomas in younger women } \\
\text { after breast cancer treatment }\end{array}$ & Mezencev $^{14}$ \\
\hline SEER & Glioma & 58,700 & $1975-2016$ & Prognostic factor: age & Zhou $^{124}$ \\
\hline SEER & Glioma & 22,427 & $1977-2000$ & $\begin{array}{l}\text { Prognostic factors: age, gender, year of } \\
\text { diagnosis; diagnostic factors: age, gender, } \\
\text { later year of diagnosis }\end{array}$ & Hess $^{16}$ \\
\hline SEER & Glioma & 24,340 & 1992-2007 & $\begin{array}{l}\text { Diagnostic factors: age, race (non-Hispanic } \\
\text { White) }\end{array}$ & Dubrow $^{13}$ \\
\hline SEER & Glioma & 1,067 & 1994-2002 & $\begin{array}{l}\text { Median OS 9, } 4,57 \text { and } 9 \text { months for low- } \\
\text { grade glioma, AA, OG, and AO, respec- } \\
\text { tively; prognostic factors: age, WHO grade }\end{array}$ & Iwamoto 25 \\
\hline SEER & Glioma & 9,385 & 1999-2010 & & Dong $^{24}$ \\
\hline
\end{tabular}


Table 1 (Continued)

\begin{tabular}{|c|c|c|c|c|c|}
\hline $\begin{array}{l}\text { Database } \\
\text { used }\end{array}$ & $\begin{array}{l}\text { Patient } \\
\text { pathology }\end{array}$ & $\begin{array}{l}\text { Sample } \\
\text { size }\end{array}$ & Study year & Study findings & Reference \\
\hline & & & & $\begin{array}{l}\text { Improved survival for grade II and III glio- } \\
\text { mas over time }\end{array}$ & \\
\hline SEER & Glioma & 24,230 & $2000-2006$ & $\begin{array}{l}\text { Greater incidence gliomas in counties with } \\
\text { higher socioeconomic status }\end{array}$ & Plascak $^{19}$ \\
\hline SEER & Glioma & 617 & $2000-2014$ & Prognostic factor: marital status & Long $^{26}$ \\
\hline SEER & Glioma & 244,808 & $2000-2014$ & $\begin{array}{l}\text { Prognostic factor: race; non-Hispanic } \\
\text { whites showed lower survival }\end{array}$ & Ostrom $^{1}$ \\
\hline SEER & Glioma & 43,324 & $2000-2016$ & Prognostic factor: marital status & $\mathrm{Xie}^{28}$ \\
\hline SEER & Glioma & 50,170 & $2003-2012$ & Prognostic factor: socioeconomic status & $\operatorname{Deb}^{23}$ \\
\hline SEER & Glioma & 10,591 & $2005-2013$ & $\begin{array}{l}\text { Prognostic factor: age, race (White), non- } \\
\text { cerebral tumor sites }\end{array}$ & $\operatorname{Sun}^{51}$ \\
\hline $\begin{array}{l}\text { SEER; Medicare } \\
\text { database }\end{array}$ & Glioma & 1,958 & 1991-1999 & Prognostic factors: age, EOR, RT & Barnholtz-Sloan 50 \\
\hline NCDB & Glioma & 1,029 & $2004-2012$ & $\begin{array}{l}\text { No difference between single- and multi- } \\
\text { agent chemotherapy }\end{array}$ & Haque $^{125}$ \\
\hline NCDB & Glioma & 2,253 & $2004-2013$ & Prognostic factor: RCT & $\mathrm{Wu}^{27}$ \\
\hline NCDB & Glioma & 5,539 & $2004-2014$ & Prognostic factor: high-volume facility & Zhu $^{29}$ \\
\hline NCDB & Glioma & 1,952 & $2010-2012$ & Prognostic factor: histology, RT & Youseff ${ }^{36}$ \\
\hline NCDB & Glioma & 1,032 & $2010-2013$ & Prognostic factor: WHO grade, tumor size & Jairam $^{32}$ \\
\hline SEER & Glioma & 2,009 & $1973-2001$ & $\begin{array}{l}\text { Prognostic factor: gender (female), age, } \\
\text { race (White), WHO grade, later year of } \\
\text { diagnosis; improved survival over time }\end{array}$ & Claus $^{30}$ \\
\hline SEER & Glioma & 2,825 & $1973-2011$ & $\begin{array}{l}\text { Indicated lack of OS improvement for LGG; } \\
\text { suggested importance of molecular } \\
\text { markers }\end{array}$ & Claus $^{31}$ \\
\hline SEER & Glioma & 42,622 & $2000-2013$ & Evaluated primary and secondary GBM & Nguyen $^{34}$ \\
\hline SEER & Glioma & 5,037 & $2001-2006$ & $\begin{array}{l}\text { Patients diagnosed with LGG } 17 \mathrm{X} \text { more } \\
\text { likely to die than general population; older } \\
\text { patients with LGG } 31 \mathrm{X} \text { more likely to die } \\
\text { than young adults in first year }\end{array}$ & $\mathrm{Smoll}^{35}$ \\
\hline SEER & Glioma & 3,732 & $2004-2013$ & $\begin{array}{l}\text { Prognostic factors: age, marital status, tu- } \\
\text { mor site, histological type, tumor size, } \\
\text { surgery, and sex }\end{array}$ & Zhao $^{22}$ \\
\hline SEER & Glioma & 561 & $2006-2012$ & Prognostic factor: EOR & Diaz-Aguilar $^{126}$ \\
\hline SEER, TCGA & Glioma & 1,278 & $1999-2016$ & $\begin{array}{l}\text { Overlap of risk genes in Alzheimer disease } \\
\text { and gliomas (TREM2, SPI1, CD33, and } \\
\text { INPP5D) }\end{array}$ & Lehrer $^{33}$ \\
\hline NCDB & Grade II glioma & 1,054 & $2004-2013$ & $\begin{array}{l}\text { RCT not associated with higher survival in } \\
\text { comparison to chemotherapy alone }\end{array}$ & Jhaveri $^{17}$ \\
\hline SEER & Grade II glioma & 1,980 & $1999-2010$ & $\begin{array}{l}\text { Prognostic factors: frontal lobe location, } \\
\text { EOR, age }\end{array}$ & Alattar $^{5}$ \\
\hline SEER & Grade II glioma & 4,113 & $1999-2010$ & Prognostic factors: EOR & Schupper $^{6}$ \\
\hline CSP & HGG & 2,743 & $1990-2000$ & $\begin{array}{l}\text { 5-year OS 6\%; median OS } 6.6 \text { months; } \\
\text { improved survival over time; prognostic } \\
\text { factors: age, WHO grade, tumor site, pri- } \\
\text { mary treatment, year of treatment, aca- } \\
\text { demic hospital }\end{array}$ & Tsao-Wei ${ }^{45}$ \\
\hline PMSI & HGG & 1,659 & $2010-2018$ & $\begin{array}{l}\text { Median OS was } 1.4 \text { years; prognostic fac- } \\
\text { tors: carmustine wafer, age, gender, recur- } \\
\text { rent disease }\end{array}$ & Champeaux $^{46}$ \\
\hline $\begin{array}{l}\text { MarketScan and Medi- } \\
\text { care supplemental } \\
\text { health claims database }\end{array}$ & HGG & 2,157 & $2009-2015$ & $\begin{array}{l}\text { No significant association between use of } \\
\text { BEV and occurrence of thromboembolic } \\
\text { events }\end{array}$ & $\operatorname{Lee}^{53}$ \\
\hline
\end{tabular}


112 Utility of Administrative Databases and Big Data on Understanding Glioma Treatment Owens et al.

Table 1 (Continued)

\begin{tabular}{|c|c|c|c|c|c|}
\hline $\begin{array}{l}\text { Database } \\
\text { used }\end{array}$ & $\begin{array}{l}\text { Patient } \\
\text { pathology }\end{array}$ & $\begin{array}{l}\text { Sample } \\
\text { size }\end{array}$ & Study year & Study findings & Reference \\
\hline SEER & HGG & 154 & $1973-2013$ & $\begin{array}{l}\text { Median OS } 10 \text { months; prognostic factors: } \\
\text { non-brainstem location, RT }\end{array}$ & Maxwell ${ }^{47}$ \\
\hline SEER & HGG & 353 & $1973-2015$ & $\begin{array}{l}\text { Prognostic factors: age; no tumor-related } \\
\text { characteristics were associated with } \\
\text { survival }\end{array}$ & Yang $^{49}$ \\
\hline SEER & HGG & 14,461 & $1998-2007$ & Prognostic factor: WHO grade, RT & Rusthoven ${ }^{48}$ \\
\hline SEER & HGG & 3,706 & $2000-2013$ & $\begin{array}{l}\text { Median OS } 14.3 \text { month; 5-year OS } 6.2 \% \text {; } \\
\text { prognostic factor: age, location (unilateral), } \\
\text { EOR, RT }\end{array}$ & $\mathrm{Xia}^{127}$ \\
\hline ACS-NSQIP & $\begin{array}{l}\text { Mix of malignant } \\
\text { brain tumor }\end{array}$ & 4,407 & $2007-2012$ & $\begin{array}{l}\text { Perioperative steroid use associated with } \\
\text { shorter hospitalization and increased read- } \\
\text { mission; no adverse events with steroid use }\end{array}$ & Alan $^{54}$ \\
\hline CBTRUS & Oligodendroglioma & & $2000-2013$ & $\begin{array}{l}\text { OD and AOD showed decreased incidence } \\
\text { over time }\end{array}$ & Achey $^{7}$ \\
\hline SEER & Oligodendroglioma & 762 & $1973-2012$ & $\begin{array}{l}\text { Prognostic factors: age, gender, race; im- } \\
\text { proved survival over time }\end{array}$ & Furst $^{12}$ \\
\hline SEER & Oligodendroglioma & 7,001 & $1973-2013$ & Prognostic factor age & Lau $^{11}$ \\
\hline SEER & Oligodendroglioma & 3,406 & $1999-2010$ & $\begin{array}{l}\text { Prognostic factors: EOR; limited benefit for } \\
\text { GTR in OG compared to AA or GBM }\end{array}$ & Alattar $^{8}$ \\
\hline SEER & Oligodendroglioma & 3,880 & 1999-2012 & Improved survival over time & Brandel $^{9}$ \\
\hline SEER & Oligodendroglioma & 3,135 & $2004-2013$ & Prognostic factors: EOR & Kinslow ${ }^{10}$ \\
\hline
\end{tabular}

Abbreviations: AA anaplastic astrocytoma; AO, anaplastic oligodendroglioma; ACS-NSQIP, American College of Surgeons National Surgical Quality Improvement Program database; BZM, bevacizumab; CA, California; CBTRUS, Central Brain Tumor Registry of the United States; CAD, coronary artery disease; CCR, California Cancer Registry; CHF, congestive heart failure; CSP, Cancer Surveillance Program; EOR, extent-of-resection; FCDS, Florida Cancer Data Registry; GBM, glioblastoma multiforme; GTR, gross total resection; HGG, high-grade glioma; LGG, low-grade glioma; NCDB, National Cancer Database; NIS, National Inpatient Sample; OS, overall survival; PMSI, French medico-administrative national database; RT, radiotherapy; RTOG, Radiation Therapy Oncology Group; SEE, Southern and Eastern Europe Tumor Registry; SEER, Surveillance, Epidemiology and End Results Database; TCGA, The Cancer Genome Atlas; TMZ, temozolomide; UCSF, University of California - San Francisco.

AO via the NCDB database between 2004 and $2013 .{ }^{41}$ An overall 5 -year of $59.8 \%$ was seen and significant benefits in survival were seen from RCT or single-agent chemotherapy. Patients were more likely to receive adjuvant RCT if treated in later time epochs, were male, had private insurance, or had $\geq$ $\$ 63,000$ median income. While the survival benefit from AOs compared with AAs were not unexpected, differences in treatment with RCT often depended on socioeconomic factors.

HGG also represent a diverse group of studied pathologies commonly aggregated in database studies. Tsao-Wei et al showed improved survival in HGG in Los Angeles County over time between 1990 and $2000 .{ }^{45}$ In addition, this study showed relative improvement in survival of GBM compared with grade III gliomas over the same time period despite an OS of $6 \%$ at 5 -years for the study group. Several other studies regarding HGG have studied several prognostic factors including age, gender, recurrent disease, tumor location, WHO grade, primary treatment, RT, and EOR. ${ }^{28,45-52}$ Another study by Lee et al showed no association between bevacizumab use and thromboembolic events..$^{53}$ One study evaluated 4,407 patients via the NSQIP database who underwent resection of a malignant brain tumor between 2007 and 2012. This study included metastatic tumors $(n=1611)$ and malignant gliomas $(n=2796) .{ }^{54}$ Steroid use was found to decrease hospital length of stay but increase risk of readmission in an unmatched case-control analysis; however, these findings were not confirmed on a propensity matched analysis.

\section{Glioblastoma}

Studies on GBM encompass the largest topic of database analysis in gliomas. A total of 68 studies were published between 2002 and 2020 (-Table 1). Studies evaluating prognostic factors showed that age, tumor histology, molecular markers, race, sex, education, marital status, treatment in high-volume and/or urban centers, insurance status, EOR, RCT, and tumor location impacted outcomes. ${ }^{52,55-111}$ Although a few studies simply described general demographic changes of GBMs, ${ }^{112,113}$ most studied specific questions. ${ }^{52,55-91,93-110,114-116}$ Most studies also demonstrated improved survival over time, which was likely associated with an increased use of RCT, temozolomide, and adjuvant treatments, such as bevacizumab, but could not deliver more granular specifics.

The impact of facility type and location on outcome after GBM treatment has been evaluated by several studies. Aulakh et a ${ }^{55}$ evaluated 60,672 patients in the NCDB from 2004 to 2013, demonstrating that treatment in a highvolume facility, along with temozolomide treatment, 
improved survival. In fact, treatment in a high-volume center conferred a 2-month OS benefit. Another study by Hauser et $\mathrm{al}^{64}$ evaluating 89,839 patients in the NCDB from 2004 to 2013 suggested better outcomes for patients treated in academic centers, and a study by Delavar et al. ${ }^{103}$ evaluating 37,581 patients between 2001 and 2011 showed a 3-month improvement in OS for patients living in metropolitan areas compared with nonmetropolitan areas. Other studies support the impact of institution type on treatment accessibility and outcome. ${ }^{65,68}$ Nuno et al ${ }^{117}$ evaluated 1,273 patients in the SEER database between 1991 and 2007 to compare hospitals with low and high rates of readmission after GBM treatment. No differences were seen in patient outcomes such as complications, nonroutine discharge, length of stay, EOR, or OS. Thus, facility characteristics, such as patient volume and facility location, seemed to impact outcome.

Several studies have evaluated the impact on outcome of socioeconomic factors, namely, marital status, race/ethnicity, and insurance status. Several studies have shown the positive impact of marriage on outcome after GBM. $^{52,56,68,69,75,78,87,89,104,110,118}$ Several studies have shown the impact of race on outcome, primarily demonstrating that non-White races fared worse. $^{52,69,71,74,87,89,95,100,101,104,118,119}$ Aizer et al ${ }^{69}$ studied 22,777 patients between 1998 and 2007 via the SEER database and showed that factors associated with omission of RT included African-American and AsianAmerican races, unmarried status, and lower annual income. Other factors associated with reduced likelihood of RT included older age and subtotal resection/biopsy. Forst et $\mathrm{al}^{104}$ evaluated 12,437 patients via the SEER database between 2002 and 2011, showing that hospice enrollment was associated with higher education, White race, and lower median income among other factors. Studying 13,665 patients in the SEER database between 2007 and 2012, Rong et al $^{110}$ showed that insurance status highly affected prognosis. Patients who were uninsured or had Medicaid coverage were likely to be younger and unmarried and to present with larger tumors. In addition, incremental survival benefits from 2007 through 2011 were seen in insured patients but not uninsured patients.

\section{Discussion}

An evaluation of the use of administrative databases and big data in the study of gliomas demonstrated 122 studies across a wide range of pathologies and clinical questions. While we aimed to characterize the important prognostic factors seen across various geographic locales and significant time frames, there remained limitations in the granular study details important to clinical applicability. Several factors, including age, tumor grade and histology, as well as surgical EOR and adjuvant therapies, were reliably impactful on patient survival which were unsurprising. More specific patient situations or treatments that offer insight to treatment could often not be determined based on the database structure. Other prognostic factors often were not reproducible among different studies. Numerous studies show improvement in survival rates over time and loosely attribute this to improved surgical technique and optimal timing of adjuvant RCT. But the impact of specific treatment changes could not be identified from these databases. The incorporation of molecular diagnostics in the discussion of these patients was commonly lacking and multiple studies combined different pathologies or WHO grade tumors to report findings.

One of the major advantages of large databases is the ability to compare across variation of socioeconomic factors, such as race, marital status, and insurance status, which would not be possible for many individual centers which have more homogeneous patient populations. This allows tracking of outcomes in disadvantaged patients and raises awareness for the patient management. In addition, some studies have shown the improvement of survival for patients in high-volume centers or metropolitan areas, suggesting variation in care delivery. However, the major disadvantage of large databases involves higher level of detail regarding patients and tumor types. These group of studies also did not indicate avenues to improve on health care disparities despite detecting them multiple times.

Improved analysis of large administrative databases is still needed. Despite some reported benefits, large databases are generally not designed to address all clinically relevant study questions with precision. One example of this is that different studies can show contradictory results for various prognostic factors. This may be likely a result of patient heterogeneity or the statistical analysis performed. Use of traditional statistical methods have the potential to identify statistically significant but not clinically relevant findings. Improvements are needed in our ability to analyze data from large administrative databases to ensure we can answer impactful questions and recognize reproducible epidemiologic patterns. Use of uniform reporting criteria for these studies, such as the STROBE criteria, is also necessary to improve the quality of these studies.

\section{Conclusions}

This study helped identify databases involved in the understanding of treatment for various gliomas. Descriptive information regarding the involved databases is provided. Consistent prognostic factors included age, tumor grade, histology, and EOR. While improvement in survival was seen over time, it was unclear which treatments specifically impacted this. In addition, marked socioeconomic, and racial disparities in health care persisted over time for a variety of pathologies. Administrative databases were also limited in integrating updates in molecular tumor subtypes. The application of insights from research databases to impact patient care remains inadequate.

Funding

None. 
Conflict of Interest

None declared.

\section{References}

1 Ostrom QT, Cote DJ, Ascha M, Kruchko C, Barnholtz-Sloan JS. Adult glioma incidence and survival by race or ethnicity in the United States From 2000 to 2014. JAMA Oncol 2018;4(09): 1254-1262

2 Karsy M, Neil JA, Guan J, Mahan MA, Colman H, Jensen RL. A practical review of prognostic correlations of molecular biomarkers in glioblastoma. Neurosurg Focus 2015;38(03):E4

3 Oravec CS, Motiwala M, Reed K, et al. Big data research in neurosurgery: a critical look at this popular new study design. Neurosurgery 2018;82(05):728-746

4 Jhaveri J, Liu Y, Chowdhary M, et al. Is less more? Comparing chemotherapy alone with chemotherapy and radiation for highrisk grade 2 glioma: an analysis of the National Cancer Data Base. Cancer 2018;124(06):1169-1178

5 Alattar AA, Carroll KT, Bryant AK, et al. Prognostic importance of age, tumor location, and tumor grade in grade II astrocytomas: An Integrated Analysis of the Cancer Genome Atlas and the Surveillance, Epidemiology, and End Results Database. World Neurosurg 2019;121:e411-e418

6 Schupper AJ, Hirshman BR, Carroll KT, Ali MA, Carter BS, Chen CC. Effect of Gross Total Resection in World Health Organization Grade II Astrocytomas: SEER-Based Survival Analysis. World Neurosurg 2017;103:741-747

7 Achey RL, Khanna V, Ostrom QT, Kruchko C, Barnholtz-Sloan JS. Incidence and survival trends in oligodendrogliomas and anaplastic oligodendrogliomas in the United States from 2000 to 2013: a CBTRUS Report. J Neurooncol 2017;133(01):17-25

8 Alattar AA, Brandel MG, Hirshman BR, et al. Oligodendroglioma resection: a Surveillance, Epidemiology, and End Results (SEER) analysis. J Neurosurg 2018;128(04):1076-1083

9 Brandel MG, Alattar AA, Hirshman BR, et al. Survival trends of oligodendroglial tumor patients and associated clinical practice patterns: a SEER-based analysis. J Neurooncol 2017;133(01): 173-181

10 Kinslow CJ, Garton ALA, Rae AI, et al. Extent of resection and survival for oligodendroglioma: a U.S. population-based study. J Neurooncol 2019;144(03):591-601

11 Lau CS, Mahendraraj K, Chamberlain RS. Oligodendrogliomas in pediatric and adult patients: an outcome-based study from the Surveillance, Epidemiology, and End Result database. Cancer Manag Res 2017;9:159-166

12 Furst T, Hoffman H, Chin LS. All-cause and tumor-specific mortality trends in geriatric oligodendroglioma (OG) patients: A surveillance, epidemiology, and end results (SEER) analysis. J Clin Neurosci 2020;73:94-100

13 Dubrow R, Darefsky AS. Demographic variation in incidence of adult glioma by subtype, United States, 1992-2007. BMC Cancer 2011;11:325

14 Mezencev R. Epidemiology of gliomas in women diagnosed with breast cancer supports the protective role of estrogenic exposure. Bratisl Lek Listy 2018;119(08):463-468

15 Persaud-Sharma D, Burns J, Trangle J, et al. Demographic variation in the frequency of gliomas in Florida. Medicina (Kaunas) 2019;55(01):E5

16 Hess KR, Broglio KR, Bondy ML. Adult glioma incidence trends in the United States, 1977-2000. Cancer 2004;101(10):2293-2299

17 Jhaveri J, Cheng E, Tian S, et al. Proton vs. Photon Radiation Therapy for Primary Gliomas: An Analysis of the National Cancer Data Base. Front Oncol 2018;8:440

$18 \mathrm{Li} \mathrm{K,} \mathrm{Lu} \mathrm{D,} \mathrm{Guo} \mathrm{Y,} \mathrm{et} \mathrm{al.} \mathrm{Trends} \mathrm{and} \mathrm{patterns} \mathrm{of} \mathrm{incidence} \mathrm{of} \mathrm{diffuse}$ glioma in adults in the United States, 1973-2014. Cancer Med 2018;7(10):5281-5290
19 Plascak JJ, Fisher JL. Area-based socioeconomic position and adult glioma: a hierarchical analysis of surveillance epidemiology and end results data. PLoS One 2013;8(04):e60910

20 Gittleman H, Ostrom QT, Stetson LC, et al. Sex is an important prognostic factor for glioblastoma but not for nonglioblastoma. Neurooncol Pract 2019;6(06):451-462

21 Samaan MC, Akhtar-Danesh N. The impact of age and race on longevity in pediatric astrocytic tumors: A population-based study. Pediatr Blood Cancer 2015;62(09):1567-1571

22 Zhao YY, Chen SH, Hao Z, Zhu HX, Xing ZL, Li MH. A nomogram for predicting individual prognosis of patients with low-grade glioma. World Neurosurg 2019;130:e605-e612

23 Deb S, Pendharkar AV, Schoen MK, Altekruse S, Ratliff J, Desai A. The effect of socioeconomic status on gross total resection, radiation therapy and overall survival in patients with gliomas. J Neurooncol 2017;132(03):447-453

24 Dong X, Noorbakhsh A, Hirshman BR, et al. Survival trends of grade I, II, and III astrocytoma patients and associated clinical practice patterns between 1999 and 2010: A SEER-based analysis. Neurooncol Pract 2016;3(01):29-38

25 Iwamoto FM, Reiner AS, Nayak L, Panageas KS, Elkin EB, Abrey LE. Prognosis and patterns of care in elderly patients with glioma. Cancer 2009;115(23):5534-5540

26 Long S, Li M, Ou S, Li G. The effect of marital status on glioma patient survival: analysis of 617 cases: A SEER-based study. Medicine (Baltimore) 2018;97(52):e13900

$27 \mathrm{Wu}$ J, Neale N, Huang Y, et al. Comparison of Adjuvant Radiation Therapy Alone and Chemotherapy Alone in Surgically Resected Low-Grade Gliomas: Survival Analyses of 2253 Cases from the National Cancer Data Base. World Neurosurg 2018;112: e812-e822

28 Xie JC, Yang S, Liu XY, Zhao YX. Marital status is associated with survival of patients with astrocytoma. J Clin Neurosci 2018; 56:79-87

29 Zhu P, Du XL, Blanco AI, et al. Impact of facility type and volume in low-grade glioma outcomes. J Neurosurg (e-pub ahead of print). Doi: 10.3171/2019.6.JNS19409

30 Claus EB, Black PM. Survival rates and patterns of care for patients diagnosed with supratentorial low-grade gliomas: data from the SEER program, 1973-2001. Cancer 2006;106(06):1358-1363

31 Claus EB, Walsh KM, Wiencke JK, et al. Survival and low-grade glioma: the emergence of genetic information. Neurosurg Focus 2015;38(01):E6

32 Jairam V, Kann BH, Park HS, et al. Defining an Intermediate-risk Group for Low-grade Glioma: A National Cancer Database Analysis. Anticancer Res 2019;39(06):2911-2918

33 Lehrer S. Glioma and Alzheimer's disease. J Alzheimers Dis Rep 2018;2(01):213-218

34 Nguyen HS, Best B, Doan NB, et al. Glioblastoma in the setting of prior lower grade gliomas - insights from SEER database. Oncotarget 2018;9(70):33271-33277

35 Smoll NR, Gautschi OP, Schatlo B, Schaller K, Weber DC. Relative survival of patients with supratentorial low-grade gliomas. Neuro-oncol 2012;14(08):1062-1069

36 Youssef I, Lee A, Garay EL, Becker DJ, Schreiber D. Patterns of care and outcomes of postoperative radiation for low-grade gliomas in United States hospitals. J Clin Neurosci 2018;58:124-129

37 Missios S, Kalakoti P, Nanda A, Bekelis K. Craniotomy for glioma resection: a predictive model. World Neurosurg 2015;83(06): 957-964

38 Mukherjee D, Sarmiento JM, Nosova K, et al. Effectiveness of radiotherapy for elderly patients with anaplastic gliomas. J Clin Neurosci 2014;21(05):773-778

39 Padwal JA, Dong X, Hirshman BR, Hoi-Sang U, Carter BS, Chen CC. Superior efficacy of gross total resection in anaplastic astrocytoma patients relative to glioblastoma patients. World Neurosurg 2016;90:186-193 
40 Shin JY, Diaz AZ. Anaplastic astrocytoma: prognostic factors and survival in 4807 patients with emphasis on receipt and impact of adjuvant therapy. J Neurooncol 2016;129(03):557-565

41 Shin JY, Diaz AZ. Utilization and impact of adjuvant therapy in anaplastic oligodendroglioma: an analysis on 1692 patients. J Neurooncol 2016;129(03):567-575

42 Shin JY, Yoon JK, Diaz AZ. Racial disparities in anaplastic oligodendroglioma: An analysis on 1643 patients. J Clin Neurosci 2017;37:34-39

43 Smoll NR, Hamilton B. Incidence and relative survival of anaplastic astrocytomas. Neuro-oncol 2014;16(10):1400-1407

44 Zhao YY, Wan QS, Hao Z, Zhu HX, Xing ZL, Li MH. Clinical nomogram for predicting the survival of patients with cerebral anaplastic gliomas. Medicine (Baltimore) 2020;99(10):e19416

45 Tsao-Wei DD, Hu J, Groshen SG, Chamberlain MC. Conditional survival of high-grade glioma in Los Angeles County during the year 1990-2000. J Neurooncol 2012;110(01):145-152

46 Champeaux C, Weller J. Implantation of carmustine wafers (Gliadel) for high-grade glioma treatment. A 9-year nationwide retrospective study. J Neurooncol 2020;147(01):159-169

47 Maxwell R, Luksik AS, Garzon-Muvdi T, et al. Population-based study determining predictors of cancer-specific mortality and survival in pediatric high-grade brainstem glioma. World Neurosurg 2018;119:e1006-e1015

48 Rusthoven CG, Carlson JA, Waxweiler TV, et al. The impact of adjuvant radiation therapy for high-grade gliomas by histology in the United States population. Int J Radiat Oncol Biol Phys 2014; 90(04):894-902

49 Yang W, Xu T, Garzon-Muvdi T, Jiang C, Huang J, Chaichana KL. Survival of Ventricular and Periventricular High-Grade Gliomas: A Surveillance, Epidemiology, and End Results Program-Based Study. World Neurosurg 2018;111:e323-e334

50 Barnholtz-Sloan JS, Williams VL, Maldonado JL, et al. Patterns of care and outcomes among elderly individuals with primary malignant astrocytoma. J Neurosurg 2008;108(04):642-648

51 Sun Y, Xiong ZY, Yan PF, Jiang LL, Nie CS, Wang X. Characteristics and prognostic factors of age-stratified high-grade intracranial glioma patients: a population-based analysis. Bosn J Basic Med Sci 2019;19(04):375-383

52 Xie JC, Yang S, Liu XY, Zhao YX. Effect of marital status on survival in glioblastoma multiforme by demographics, education, economic factors, and insurance status. Cancer Med 2018;7 (08):3722-3742

53 Lee I, Adimadhyam S, Nutescu EA, et al. Bevacizumab use and the risk of arterial and venous thromboembolism in patients with high-grade gliomas: a nested case-control study. Pharmacotherapy 2019;39(09):921-928

54 Alan N, Seicean A, Seicean S, Neuhauser D, Benzel EC, Weil RJ. Preoperative steroid use and the incidence of perioperative complications in patients undergoing craniotomy for definitive resection of a malignant brain tumor. J Clin Neurosci 2015;22 (09):1413-1419

55 Aulakh S, DeDeo MR, Free J, et al. Survival trends in glioblastoma and association with treating facility volume. J Clin Neurosci 2019;68:271-274

56 Dressler EV, Liu M, Garcia CR, et al. Patterns and disparities of care in glioblastoma. Neurooncol Pract 2019;6(01):37-46

57 Glaser SM, Dohopolski MJ, Balasubramani GK, Flickinger JC, Beriwal S. Glioblastoma multiforme (GBM) in the elderly: initial treatment strategy and overall survival. J Neurooncol 2017;134(01):107-118

58 Mak KS, Agarwal A, Qureshi MM, Truong MT. Hypofractionated short-course radiotherapy in elderly patients with glioblastoma multiforme: an analysis of the National Cancer Database. Cancer Med 2017;6(06):1192-1200

59 Malakhov N, Lee A, Garay E, Becker DJ, Schreiber D. Patterns of care and outcomes for glioblastoma in patients with poor performance status. J Clin Neurosci 2018;52:66-70
60 Lee A, Malakhov N, Sheth N, Wang A, Han P, Schreiber D. Patterns of care and outcomes of chemoradiation versus radiation alone for MGMT promoter unmethylated glioblastoma. Clin Neurol Neurosurg 2018;170:127-131

61 Lee A, Youssef I, Osborn VW, Safdieh J, Becker DJ, Schreiber D. The utilization of MGMT promoter methylation testing in United States hospitals for glioblastoma and its impact on prognosis. J Clin Neurosci 2018;51:85-90

62 Buszek SM, Al Feghali KA, Elhalawani H, Chevli N, Allen PK, Chung C. Optimal Timing of Radiotherapy Following Gross Total or Subtotal Resection of Glioblastoma: A Real-World Assessment using the National Cancer Database. Sci Rep 2020;10(01): 4926

63 Haque W, Thong Y, Verma V, Rostomily R, Brian Butler E, Teh BS. Patterns of management and outcomes of unifocal versus multifocal glioblastoma. J Clin Neurosci 2020;74:155-159

64 Hauser A, Dutta SW, Showalter TN, Sheehan JP, Grover S, Trifiletti DM. Impact of academic facility type and volume on postsurgical outcomes following diagnosis of glioblastoma. J Clin Neurosci 2018;47:103-110

65 Rhome R, Fisher R, Hormigo A, Parikh RR. Disparities in receipt of modern concurrent chemoradiotherapy in glioblastoma. J Neurooncol 2016;128(02):241-250

66 Kole AJ, Park HS, Yeboa DN, et al. Concurrent chemoradiotherapy versus radiotherapy alone for "biopsy-only" glioblastoma multiforme. Cancer 2016;122(15):2364-2370

67 Trifiletti DM, Alonso C, Grover S, Fadul CE, Sheehan JP, Showalter TN. Prognostic implications of extent of resection in glioblastoma: analysis from a large database. World Neurosurg 2017;103:330-340

68 Wegner RE, Abel S, Horne ZD, et al. National trends in radiation dose escalation for glioblastoma. Radiat Oncol J 2019;37(01): $13-21$

69 Aizer AA, Ancukiewicz M, Nguyen PL, Shih HA, Loeffler JS, Oh KS. Underutilization of radiation therapy in patients with glioblastoma: predictive factors and outcomes. Cancer 2014; 120(02):238-243

70 Al-Husseini MJ, Saad AM, El-Shewy KM, et al. Prior malignancy impact on survival outcomes of glioblastoma multiforme; population-based study. Int J Neurosci 2019;129(05):447-454

71 Barnholtz-Sloan JS, Maldonado JL, Williams VL, et al. Racial/ethnic differences in survival among elderly patients with a primary glioblastoma. J Neurooncol 2007;85(02):171-180

72 Bartek J Jr, Alattar AA, Dhawan S, et al. Receipt of brachytherapy is an independent predictor of survival in glioblastoma in the Surveillance, Epidemiology, and End Results database. J Neurooncol 2019;145(01):75-83

73 Siker ML, Wang M, Porter K, et al. Age as an independent prognostic factor in patients with glioblastoma: a Radiation Therapy Oncology Group and American College of Surgeons National Cancer Data Base comparison. J Neurooncol 2011;104 (01):351-356

74 Thumma SR, Fairbanks RK, Lamoreaux WT, et al. Effect of pretreatment clinical factors on overall survival in glioblastoma multiforme: a Surveillance Epidemiology and End Results (SEER) population analysis. World J Surg Oncol 2012;10:75

75 Walker GV, Li J, Mahajan A, et al. Decreasing radiation therapy utilization in adult patients with glioblastoma multiforme: a population-based analysis. Cancer 2012;118(18):4538-4544

76 Yusuf MB, Gaskins J, Amsbaugh MJ, Woo S, Burton E. Survival impact of prolonged postoperative radiation therapy for patients with glioblastoma treated with combined-modality therapy. Neurooncol Pract 2019;6(02):112-123

77 Zinn PO, Colen RR, Kasper EM, Burkhardt JK. Extent of resection and radiotherapy in GBM: A 1973 to 2007 surveillance, epidemiology and end results analysis of 21,783 patients. Int J Oncol 2013;42(03):929-934 
78 Chang SM, Barker FG II. Marital status, treatment, and survival in patients with glioblastoma multiforme: a population based study. Cancer 2005;104(09):1975-1984

79 Chen JH, Huang CY, Lee YC, et al. Comparative cost analysis for the surgical and endovascular treatment of ruptured intracranial aneurysms in Taiwan: a nationwide population-based cohort study. World Neurosurg 2018; 116:e485-e490

80 Darefsky AS, King JT Jr, Dubrow R. Adult glioblastoma multiforme survival in the temozolomide era: a populationbased analysis of Surveillance, Epidemiology, and End Results registries. Cancer 2012;118(08):2163-2172

81 Iwamoto FM, Reiner AS, Panageas KS, Elkin EB, Abrey LE. Patterns of care in elderly glioblastoma patients. Ann Neurol 2008;64 (06):628-634

82 Adams H, Adams HH, Jackson C, Rincon-Torroella J, Jallo GI, Quiñones-Hinojosa A. Evaluating extent of resection in pediatric glioblastoma: a multiple propensity score-adjusted population-based analysis. Childs Nerv Syst 2016;32(03): 493-503

83 Johnson DR, Ma DJ, Buckner JC, Hammack JE. Conditional probability of long-term survival in glioblastoma: a population-based analysis. Cancer 2012;118(22):5608-5613

84 Johnson DR, O'Neill BP. Glioblastoma survival in the United States before and during the temozolomide era. J Neurooncol 2012;107(02):359-364

85 Lam S, Lin Y, Zinn P, Su J, Pan IW. Patient and treatment factors associated with survival among pediatric glioblastoma patients: A Surveillance, Epidemiology, and End Results study. J Clin Neurosci 2018;47:285-293

86 Noorbakhsh A, Tang JA, Marcus LP, et al. Gross-total resection outcomes in an elderly population with glioblastoma: a SEERbased analysis. J Neurosurg 2014;120(01):31-39

87 Pan IW, Ferguson SD, Lam S. Patient and treatment factors associated with survival among adult glioblastoma patients: A USA population-based study from 2000-2010. J Clin Neurosci 2015;22(10):1575-1581

88 Porter AB, Lachance DH, Johnson DR. Socioeconomic status and glioblastoma risk: a population-based analysis. Cancer Causes Control 2015;26(02):179-185

89 Shah BK, Bista A, Sharma S. Survival trends in elderly patients with glioblastoma in the United States: a population-based study. Anticancer Res 2016;36(09):4883-4886

90 Tian M, Ma W, Chen Y, et al. Impact of gender on the survival of patients with glioblastoma. Biosci Rep 2018;38(06):BSR20180752

91 Wachtel MS, Yang S. Odds of death after glioblastoma diagnosis in the United States by chemotherapeutic era. Cancer Med 2014; 3(03):660-666

92 Zhu P, Du XL, Lu G, Zhu JJ. Survival benefit of glioblastoma patients after FDA approval of temozolomide concomitant with radiation and bevacizumab: A population-based study. Oncotarget 2017;8(27):44015-44031

93 Aneja S, Khullar D, Yu JB. The influence of regional health system characteristics on the surgical management and receipt of post operative radiation therapy for glioblastoma multiforme. J Neurooncol 2013;112(03):393-401

94 Koshy M, Villano JL, Dolecek TA, et al. Improved survival time trends for glioblastoma using the SEER 17 population-based registries. J Neurooncol 2012;107(01):207-212

95 Shabihkhani M, Telesca D, Movassaghi $M$, et al. Incidence, survival, pathology, and genetics of adult Latino Americans with glioblastoma. J Neurooncol 2017;132(02):351-358

96 Shu C, Yan X, Zhang X, Wang Q, Cao S, Wang J. Tumor-induced mortality in adult primary supratentorial glioblastoma multiforme with different age subgroups. Future Oncol 2019; 15(10):1105-1114

97 Smoll NR, Schaller K, Gautschi OP. The cure fraction of glioblastoma multiforme. Neuroepidemiology 2012;39(01): 63-69
98 Arvold ND, Cefalu M, Wang Y, Zigler C, Schrag D, Dominici F. Comparative effectiveness of radiotherapy with vs. without temozolomide in older patients with glioblastoma. J Neurooncol 2017;131(02):301-311

99 Arvold ND, Wang Y, Zigler C, Schrag D, Dominici F. Hospitalization burden and survival among older glioblastoma patients. Neuro-oncol 2014;16(11):1530-1540

100 Barnholtz-Sloan JS, Sloan AE, Schwartz AG. Racial differences in survival after diagnosis with primary malignant brain tumor. Cancer 2003;98(03):603-609

101 Bohn A, Braley A, Rodriguez de la Vega P, Zevallos JC, Barengo NC. The association between race and survival in glioblastoma patients in the US: a retrospective cohort study. PLoS One 2018;13(06):e0198581

102 Davies J, Reyes-Rivera I, Pattipaka T, et al. Survival in elderly glioblastoma patients treated with bevacizumab-based regimens in the United States. Neurooncol Pract 2018;5(04):251-261

103 Delavar A, Al Jammal OM, Maguire KR, Wali AR, Pham MH. The impact of rural residence on adult brain cancer survival in the United States. J Neurooncol 2019;144(03):535-543

104 Forst D, Adams E, Nipp R, et al. Hospice utilization in patients with malignant gliomas. Neuro-oncol 2018;20(04):538-545

105 Johnson DR, Leeper HE, Uhm JH. Glioblastoma survival in the United States improved after Food and Drug Administration approval of bevacizumab: a population-based analysis. Cancer 2013;119(19):3489-3495

106 Ladomersky E, Scholtens DM, Kocherginsky M, et al. The coincidence between increasing age, immunosuppression, and the incidence of patients with glioblastoma. Front Pharmacol 2019;10:200

107 Marcus LP, McCutcheon BA, Noorbakhsh A, et al. Incidence and predictors of 30-day readmission for patients discharged home after craniotomy for malignant supratentorial tumors in California (1995-2010). J Neurosurg 2014;120(05):1201-1211

108 Pendharkar AV, Rezaii PG, Ho AL, Sussman ES, Li G, Desai AM. Functional mapping for glioma surgery: a propensity-matched analysis of outcomes and cost. World Neurosurg 2020;137: e328-e335

109 Rahmani R, Tomlinson SB, Santangelo G, et al. Risk factors associated with early adverse outcomes following craniotomy for malignant glioma in older adults. J Geriatr Oncol 2020;11 (04):694-700

110 Rong X, Yang W, Garzon-Muvdi T, et al. Influence of insurance status on survival of adults with glioblastoma multiforme: A population-based study. Cancer 2016;122(20):3157-3165

111 Huang J, Samson P, Perkins SM, et al. Impact of concurrent chemotherapy with radiation therapy for elderly patients with newly diagnosed glioblastoma: a review of the National Cancer Data Base. J Neurooncol 2017;131(03):593-601

112 Nguyen HS, Doan NB, Gelsomino M, et al. Management and survival trends for adult patients with malignant gliomas in the setting of multiple primary tumors: a population based analysis. J Neurooncol 2019;141(01):213-221

113 Zada G, Bond AE, Wang YP, Giannotta SL, Deapen D. Incidence trends in the anatomic location of primary malignant brain tumors in the United States: 1992-2006. World Neurosurg 2012;77(3-4):518-524

114 Huang LE, Cohen AL, Colman H, Jensen RL, Fults DW, Couldwell WT. IGFBP2 expression predicts IDH-mutant glioma patient survival. Oncotarget 2017;8(01):191-202

115 Chen LF, Yang Y, Ma XD, et al. Optimizing the extent of resection and minimizing the morbidity in insular high-grade glioma surgery by high-field intraoperative MRI guidance. Turk Neurosurg 2017;27(05):696-706

116 Li X, Li Y, Cao Y, et al. Risk of subsequent cancer among pediatric, adult and elderly patients following a primary diagnosis of glioblastoma multiforme: a population-based study of the SEER database. Int J Neurosci 2017;127(11):1005-1011 
117 Nuño M, Ly D, Mukherjee D, Ortega A, Black KL, Patil CG. Quality of surgical care and readmission in elderly glioblastoma patients. Neurooncol Pract 2014;1(02):33-39

118 Wang W. Increased incidence of second primary malignancy in patients with malignant astrocytoma: a population-based study. Biosci Rep 2019;39(06):BSR20181968

$119 \mathrm{Xu} \mathrm{H}, \mathrm{Chen} \mathrm{J,} \mathrm{Xu} \mathrm{H,} \mathrm{Qin} \mathrm{Z.} \mathrm{Geographic} \mathrm{variations} \mathrm{in} \mathrm{the} \mathrm{incidence} \mathrm{of}$ glioblastoma and prognostic factors predictive of overall survival in US adults from 2004-2013. Front Aging Neurosci 2017;9:352

120 Bin Abdulrahman AK, Bin Abdulrahman KA, Bukhari YR, Faqihi AM, Ruiz JG. Association between giant cell glioblastoma and glioblastoma multiforme in the United States: A retrospective cohort study. Brain Behav 2019;9(10):e01402

121 Lai R, Hershman DL, Doan T, Neugut AI. The timing of cranial radiation in elderly patients with newly diagnosed glioblastoma multiforme. Neuro-oncol 2010;12(02):190-198

122 Chen YR, Sole J, Ugiliweneza B, et al. National trends for reoperation in older patients with glioblastoma. World Neurosurg 2018;113:e179-e189
123 Chen YR, Ugiliweneza B, Burton E, Woo SY, Boakye M, Skirboll S. The effect of postoperative infection on survival in patients with glioblastoma. J Neurosurg 2017;127(04):807-811

124 Zhou X, Zhang S, Niu X, et al. Risk factors for early mortality among patients with glioma: a population-based study. World Neurosurg 2020;136:e496-e503

125 Haque W, Verma V, Butler EB, Teh BS. Patterns of care and outcomes of multi-agent versus single-agent chemotherapy as part of multimodal management of low grade glioma. J Neurooncol 2017;133(02):369-375

126 Diaz-Aguilar D, ReFaey K, Clifton W, et al. Prognostic factors and survival in low grade gliomas of the spinal cord: A populationbased analysis from 2006 to 2012. J Clin Neurosci 2019; 61:14-21

127 Xia Y, Liao W, Huang S, et al. Nomograms for Predicting the Overall and Cancer-Specific Survival of Patients with High-Grade Glioma: A Surveillance, Epidemiology, and End Results Study. Turk Neurosurg 2020;30(01):48-59 